\title{
Terapia cognitivo-conductual en las psicosis agudas.
}

Cognitive-behavioral therapy for acute psychosis.

\author{
Mª Isabel Gutiérrez López a , María Sánchez Muñoz b, Alejandra Trujillo Borrego c, Luis Sán- \\ chez Bonome d.
}

${ }^{a}$ Psicóloga clínica. CSM Cangas del Nancea. Área II. Servicio de Salud del Principado de Asturias, Espa$\tilde{n} a,{ }^{b}$ Psicóloga clínica. Hospital Universitario Virgen de la Arrixaca, Murcia. Servicio Murciano de Salud, España, ${ }^{c}$ Psicóloga Clínica. URA-HD Algeciras. Servicio Andaluz de Salud, España, ${ }^{d}$ Médico Psiquiatra. Hospital de La Línea (Cádiz). Servicio Andaluz de Salud, España.

Correspondencia: Ma Isabel Gutiérrez,López, (mariaisabelgl@hotmail.com)

Recibido: 17/06/2011; aceptado: 05/11/2011

RESUMEN: El tratamiento de las psicosis agudas era, hasta hace unos años, algo relegado al tratamiento farmacológico (a excepción del psicoanálisis). En los últimos tiempos esta tendencia ha ido cambiando y diversos estudios han puesto de manifiesto la ventaja de las intervenciones cognitivo conductuales en las fases agudas de las psicosis. A esta tendencia se ha unido un nuevo y prometedor paradigma de intervención, la intervención temprana en psicosis.

En el presente estudio se muestra una revisión bibliográfica de las investigaciones más relevantes publicadas hasta la fecha en torno a esta temática. Se incluyeron aquellos trabajos con un diseño controlado $\mathrm{y}$ aleatorio, y que garantizaban los requisitos de fiabilidad y validez.

Los estudios muestran resultados esperanzadores, en cuanto que avalan la eficacia de la TCC como medida de tratamiento. Sin embargo, todavía se hace necesario un mayor número de investigaciones que avalen estos datos.

PALABRAS CLAVE: Terapia Cognitivo-Conductual, Psicosis Aguda, Intervención Temprana.
ABSTRACT: Some years ago, the treatment for acute psychosis was something left for pharmacological treatment (except for psychoanalysis). Lately, this trend has been changing and some studies have shown the advantages of cognitive behavioural therapy in the acute psychosis. A new and promising way of intervention is merging to this trend: early intervention of psychosis.

In this study, we show a bibliographical review of the most important researches published until today on this topic. We have included the works with a random and controlled design and those which guaranteed the reliability and validity requirements.

The results of these studies are full of hope since they show the efficiency of cognitive behavioural therapy as a way of treatment. However, more research is needed to prove these facts.

KEY WORDS: Cognitive behavioural therapy, Acute psychosis, Early intervention.

\section{Introducción}

Tradicionalmente se ha entendido a los trastornos psicóticos como algo no abordable desde el punto de vista psicológico y relegado únicamente al tratamiento farmacológico. En los últimos años, esta visión ha ido cambiando, y cada vez se aboga más por un tratamiento multidisciplinar que implique el trabajo conjunto 
farmacológico y psicológico. El modelo de vulnerabilidad-estrés, que señala la interacción entre las características biológicas y psicosociales como responsable de la psicopatología psicótica, y el empuje de ciertas corrientes psicológicas que demostraban su eficacia en otros trastornos, ha contribuido ciertamente a ello.

Las técnicas psicológicas cognitivo-conductuales basan su intervención en la dotación al paciente de una serie de recursos y estrategias que le permitan afrontar su enfermedad de una manera activa. Así, el paciente dejaría de ser un recipiente pasivo de cuidados para pasar a ser un agente activo en el manejo de su enfermedad.

Si bien los primeros acercamientos al tratamiento psicólogico cognitivo-conductual de la psicosis se centraban en los síntomas negativos y/o aquellos síntomas refractarios al tratamiento psicofarmacológico, también se desarrollaron intervenciones centradas en los periodos agudos de la enfermedad.

Dentro de las intervenciones en las psicosis agudas se encuadran no sólo aquellas en las que se interviene en el brote psicótico agudo (independientemente de la evolución de la enfermedad) sino también aquellas centradas en los primeros brotes psicóticos y en los síntomas que los preceden.

Con respecto a la intervención en los primeros brotes psicóticos, actualmente numerosas investigaciones se están basando en lo que se ha dado en llamar Intervención Temprana en Psicosis $(1,2)$. Los estudios prospectivos llevados hasta la fecha indican lo que ya había propuesto Bleuler, y es que en los primeros años de la esquizofrenia es donde se produce la mayor parte del deterioro, concretamente en el periodo previo al primer brote psicótico o fase prodrómica, e inmediatamente después del primer episodio psicótico se produce el llamado efecto meseta ("plateau effect"), quedando los pacientes después más o menos estabilizados. Es por lo tanto este un período crítico en la evolución de la enfermedad en el que se forman aspectos fundamentales para el posterior curso de la enfermedad, como por ejemplo el modelo de respuesta individual a la psicosis que facilita o dificulta su posterior adaptación, el desarrollo de procesos de morbilidad secundarios como la depresión o el suicidio, y el origen de determinados componentes de implicación familiar, como la sobreimplicación o el criticismo.

Debido a esta importancia en la intervención temprana, recientes grupos de investigación vienen centrándose en el concepto de pródromo y tratan de identificar e intervenir sobre éstos con el objetivo de prevenir el comienzo de la enfermedad. Esta intervención estaría situada dentro de la prevención primaria y pretende detectar manifestaciones o factores de riesgo asociados al desarrollo posterior de una psicosis así como desarrollar formas de tratamiento que ayuden a mejorar el pronóstico y a reducir las alteraciones psicológicas, funcionales y sociales asociadas a la enfermedad.

Ésta es la conceptualización mayoritaria de los pródromos y se refiere a ellos como signos y síntomas previos a la primera manifestación de la enfermedad, sin 
ORIGINALES Y REVISIONES

embargo, estos también pueden ser asociados a la recaída (en cualquier momento de la evolución de la enfermedad). Aunque las manifestaciones clínicas de uno y otro sean sustancialmente distintas, ya que en el último caso dicha sintomatología estará influida por los síntomas residuales de la fase aguda, ésta otra conceptualización ofrece también la posibilidad de establecer un intervención temprana en psicosis.

En general, la intervención temprana debe abarcar las tres grandes áreas que interactúan a lo largo de toda la psicosis: síntomas, funcionamiento psicosocial y características psicológicas.

En el presente trabajo supone una revisión bibliográfica de aquellos estudios que evalúan la eficacia de la terapia cognitivo-conductual (TCC) aplicada a los periodos psicóticos agudos o a la intervención temprana de estos. Las características de los estudios incluidos en esta revisión se muestran en las tablas 1 y 2.

\section{Intervención sobre fases agudas}

Dentro de las intervenciones en las psicosis agudas debemos distinguir entre aquellas centradas en los primeros brotes psicóticos con aquellas en las que se interviene en el brote psicótico agudo independientemente de la evolución de la enfermedad. Las características de los estudios controlados en fase aguda se muestran en la tabla 1.

Un estudio controlado con pacientes en fase aguda $(3,4)$ comparó la TCC con terapia ocupacional y de soporte informal (TO) en 40 pacientes, 20 en cada grupo. Aproximadamente el $30 \%$ de los pacientes estaba en su primer brote psicótico. Se evaluó a los pacientes antes del tratamiento, al finalizarlo tras 8 semanas y a los 9 meses de seguimiento. La TCC incluía: 1) sesiones individuales enfocadas a cuestionar la importancia de las creencias delirantes y de las alucinaciones auditivas; 2 ) sesiones grupales, con no más de 6 pacientes, donde observaban cómo las creencias de otras personas incluyen a menudo elementos irracionales e incoherentes así como las explicaciones alternativas consideradas, también se fomentaron estrategias de afrontamiento para los síntomas psicóticos; 3) dos sesiones de trabajo con las familias donde se les explicaba como interactuar con el paciente y darle apoyo y soporte cuando se encontrase sintomático; 4) programas de actividades, tanto actividades expresivas como interpersonales, con grupos abiertos de discusión. La evaluación se llevó a cabo mediante la PAS (Psychiatric Assesment Scale) (5) y el cuestionario personal de creencias delirantes de Brett-Jones. En el seguimiento realizado a los nueve meses, la reducción de los delirios y alucinaciones era significativamente mayor en el grupo de TCC, con una disminución significativa de la convicción de las ideas delirantes y en la frecuencia de delirios y alucinaciones, 
Tabla 1

Artículos incluidos sobre fases agudas.

\begin{tabular}{|c|c|c|c|c|c|c|c|c|}
\hline ESTUDIO & MUESTRA & $\begin{array}{l}\text { GPOS DE } \\
\text { TTO }\end{array}$ & DURACIÓN & MEDIDAS & $\begin{array}{l}\text { EVALUA- } \\
\text { CIONES }\end{array}$ & RESULTADOS & $\begin{array}{l}\text { ALEA- } \\
\text { TORIO }\end{array}$ & $\begin{array}{l}\text { EVALUA- } \\
\text { DOR CIEGO }\end{array}$ \\
\hline $\begin{array}{l}\text { Drury et al } \\
(1996,2000) \\
(3,4,6)\end{array}$ & $\begin{array}{l}40 \text { pacientes } \\
\text { en fase aguda }\end{array}$ & $\begin{array}{l}\text { TCC } \\
\text { TO }\end{array}$ & \begin{tabular}{|l|} 
Sesiones indivi- \\
duales, grupales, \\
familiares y \\
programa de \\
actividades \\
durante \\
8 semanas
\end{tabular} & $\begin{array}{l}\text { SPAS } \\
\text { Cuestionario } \\
\text { de creencias } \\
\text { delirantes } \\
\text { Tiempo de } \\
\text { recuperación }\end{array}$ & $\begin{array}{l}\text { Línea base } \\
\text { Post-trata- } \\
\text { miento } \\
9 \text { meses de } \\
\text { seguimiento } \\
5 \text { años de } \\
\text { seguimiento }\end{array}$ & $\begin{array}{l}\text { TCC }>\text { TO en reducción de } \\
\text { síntomas positivos a los } 9 \\
\text { meses de seguimiento } \\
\text { TCC }>\text { TO a los } 5 \text { años de } \\
\text { seguimiento en aumento } \\
\text { de período de recaída y } \\
\text { conciencia de enfermedad }\end{array}$ & SI & NO \\
\hline $\begin{array}{l}\text { Kemps et al } \\
(1996, \\
1998)(7,9)\end{array}$ & $\begin{array}{l}47 \text { pacientes } \\
\text { con psicosis } \\
\text { funcional en } \\
\text { fase aguda }\end{array}$ & $\begin{array}{l}\text { TC } \\
\text { TAI }\end{array}$ & \begin{tabular}{|l|} 
Entre 4-6 \\
sesiones de hasta \\
una hora
\end{tabular} & \begin{tabular}{|l|} 
BPRS GAF \\
Inventario de \\
actitud hacia la \\
medicación
\end{tabular} & \begin{tabular}{|l|} 
Línea base \\
Seguimiento \\
a los 3,6 y 18 \\
meses
\end{tabular} & $\begin{array}{l}\text { TC>TAI en cumplimiento } \\
\text { y actitud hacia la medica- } \\
\text { ción y en insight. }\end{array}$ & SI & $\mathrm{NO}$ \\
\hline $\begin{array}{l}\text { O’Donell et }^{\prime} \text { al (2003) (10) }\end{array}$ & $\begin{array}{l}56 \text { pacientes } \\
\text { esquizofré- } \\
\text { nicos }\end{array}$ & $\begin{array}{l}\text { TC } \\
\text { TAI }\end{array}$ & $\begin{array}{l}5 \text { sesiones de } \\
\text { hasta una hora }\end{array}$ & $\begin{array}{l}\text { PANSS } \\
\text { SAI GAF } \\
\text { QLS }\end{array}$ & \begin{tabular}{|l|} 
Línea base \\
12 meses de \\
seguimiento
\end{tabular} & $\begin{array}{l}\mathrm{TC}=\mathrm{TAI} \text { en todas las } \\
\text { variables estudiadas }\end{array}$ & SI & SI \\
\hline $\begin{array}{l}\text { Lewis et al } \\
(2002)(15), \\
\text { Tarrier et al } \\
(2004)(16,17) \\
\text { Tarrier et al } \\
(2006)(18) \\
\text { Haddock et al } \\
(2006)(19)\end{array}$ & $\begin{array}{l}309 \text { pacientes } \\
\text { esquizofréni- } \\
\text { cos, esquizo- } \\
\text { freniformes, } \\
\text { esquizoafec- } \\
\text { tivos o } \\
\text { delirantes }\end{array}$ & $\begin{array}{l}\mathrm{TCC}+\mathrm{TR} \\
\mathrm{TA}+\mathrm{TR} \\
\mathrm{TR}\end{array}$ & \begin{tabular}{|l|} 
Sesiones de 15 \\
y 20 horas re- \\
partidas durante \\
5 semanas más \\
4 sesiones de \\
refuerzo en los 3 \\
primeros meses
\end{tabular} & $\begin{array}{l}\text { PANSS } \\
\text { PSYRATS }\end{array}$ & \begin{tabular}{|l|} 
Línea base \\
$2,3,4$ y 5 se- \\
manas durante \\
el tratamiento \\
Evaluación fi- \\
nal fase aguda \\
(entre semana \\
$6-10)$ \\
Seguimiento a \\
los 18 meses \\
\end{tabular} & $\begin{array}{l}\text { TCC }>\text { TA }>\text { TR en rapidez } \\
\text { de mejoría en la cuarta } \\
\text { semana. } \\
\text { TCC=TA=TR en mejoría } \\
\text { de conducta suicida } \\
\text { TA }>\text { TCC y TR en pacien- } \\
\text { tes menores de } 21 \text { años } \\
\text { TCC }>\text { TA y TR en pacien- } \\
\text { tes mayores de } 21 \text { años }\end{array}$ & SI & SI \\
\hline $\begin{array}{l}\text { Startup et al } \\
(2004,2005) \\
(20,25)\end{array}$ & $\begin{array}{l}90 \text { pacientes } \\
\text { hospitalizados } \\
\text { con episodios } \\
\text { agudos de } \\
\text { esquizofrenia } \\
\text { y trastornos } \\
\text { relacionados }\end{array}$ & TR & $\begin{array}{l}\text { Una sesión } \\
\text { semanal hasta un } \\
\text { máximo de } 25\end{array}$ & $\begin{array}{l}\text { SAPS } \\
\text { SANS } \\
\text { BPRS-E } \\
\text { GAF } \\
\text { SFS } \\
\text { SUS }\end{array}$ & \begin{tabular}{|l|} 
Línea base \\
6,12 y 24 \\
meses tras \\
evaluación de \\
la línea base
\end{tabular} & $\begin{array}{l}\text { TCC }>\text { TR a los } 12 \text { meses } \\
\text { en todas las variables } \\
\text { estudiadas excepto desor- } \\
\text { ganización } \\
\text { A los } 24 \text { meses TCC }>\text { TR } \\
\text { en síntomas negativos y } \\
\text { funcionamiento social }\end{array}$ & SI & $\mathrm{NO}$ \\
\hline
\end{tabular}

$\mathrm{TCC}=$ Terapia Cognitivo-Conductual; $\mathrm{TO}=$ Terapia Ocupacional y de Soporte Informal; $\mathrm{TC}=$ Terapia de Cumplimiento; $\mathrm{TAI}=$ Terapia de Asesoramiento Inespecífico; TA= Terapia de Apoyo; TR= Tratamiento Rutinario; EHS= Entrenamiento en Habilidades Sociales; PAS= Psychiatryc Assessment Scale; BPRS= Brief Psychiatric Rating Scale; GAF= Global Assessment of Functioning Scale; PANSS= Positive and Negative Syndrome Scale; SAI= Schedule for Assessment of Insignt; QLS= Escala de Heinrich; PSYRATS= Psychotic Symptom Rating Scale; SAPS= Scale for the Assessment of Positive Symptoms; SANS= Scale for the Assessment of Negative Symptoms; SFS= Social Funcioning Scale; SUS= Service Utilizatio 
Tabla 2

Estudios incluidos sobre intervención temprana en psicosis.

\begin{tabular}{|c|c|c|c|c|c|c|c|c|}
\hline ESTUDIO & MUESTRA & $\begin{array}{l}\text { GPOS DE } \\
\text { TTO }\end{array}$ & DURACIÓN & MEDIDAS & $\begin{array}{l}\text { EVALUA- } \\
\text { CIONES }\end{array}$ & RESULTADOS & $\begin{array}{l}\text { ALEA- } \\
\text { TORIO }\end{array}$ & $\begin{array}{l}\text { EVALUA- } \\
\text { DOR CIEGO }\end{array}$ \\
\hline $\begin{array}{l}\text { Jackson et } \\
\text { al (1998, } \\
2001) 29,30\end{array}$ & $\begin{array}{l}80 \text { pacientes } \\
\text { con primer } \\
\text { episodio } \\
\text { psicótico }\end{array}$ & $\begin{array}{l}\text { COPE + } \\
\text { manejo de } \\
\text { caso + TR } \\
\text { Manejo de } \\
\text { caso + TR TR }\end{array}$ & $\begin{array}{l}\text { Irregular entre } \\
\text { pacientes }\end{array}$ & \begin{tabular}{|l|} 
EM I/SO \\
SANS BPRS \\
SCLR-90 \\
BDI QLS
\end{tabular} & \begin{tabular}{|l|} 
Línea base \\
Post-tratamiento \\
12 meses de \\
seguimiento \\
\end{tabular} & $\begin{array}{l}\text { COPE }>\text { TR en comprensión } \\
\text { y adaptación de la enfer- } \\
\text { medad y en los síntomas } \\
\text { negativos. }\end{array}$ & NO & $\mathrm{NO}$ \\
\hline $\begin{array}{l}\text { Jackson et al } \\
(2005) 34\end{array}$ & $\begin{array}{l}91 \text { pacientes } \\
\text { con primer } \\
\text { episodio } \\
\text { psicótico }\end{array}$ & \begin{tabular}{|l|} 
COPE \\
TR
\end{tabular} & $\begin{array}{l}\text { Irregular entre } \\
\text { pacientes }\end{array}$ & $\begin{array}{|ll|}\text { EMS } & \text { I/SO } \\
\text { SANS } & \text { BPRS } \\
\text { SCLR-90 } \\
\text { BDI } & \text { QLS } \\
\end{array}$ & \begin{tabular}{|l|} 
Línea Base \\
6 y 12 meses \\
de seguimiento
\end{tabular} & $\begin{array}{l}\text { COPE=TR en todas las } \\
\text { variables y momentos } \\
\text { estudiados }\end{array}$ & $\mathrm{NO}$ & NO \\
\hline $\begin{array}{l}\text { Jackson et al } \\
(2008) 35\end{array}$ & $\begin{array}{l}62 \text { pacientes } \\
\text { con primer } \\
\text { episodio } \\
\text { psicótico }\end{array}$ & $\begin{array}{l}\text { ACE } \\
\text { Control }\end{array}$ & \begin{tabular}{|l|} 
Irregular \\
entre pacientes \\
(máximo de 20 \\
sesiones durante \\
14 semanas)
\end{tabular} & \begin{tabular}{|l|} 
SCID \\
BPRS \\
SANS \\
SOFAS
\end{tabular} & \begin{tabular}{|l|} 
Línea base \\
6,12 semanas \\
15 meses
\end{tabular} & $\begin{array}{l}\text { ACE }>\text { Control en términos de } \\
\text { desaparición de los síntomas } \\
\text { negativos y mayor funciona- } \\
\text { miento a mitad de tratamien- } \\
\text { to y, en menor medida, al } \\
\text { final del tratamiento } \\
\end{array}$ & SI & SI \\
\hline $\begin{array}{l}\text { Bertelsen et } \\
\text { al (2008)53 }\end{array}$ & $\begin{array}{l}547 \text { pacientes } \\
\text { con primer } \\
\text { episodio } \\
\text { psicótico }\end{array}$ & $\begin{array}{l}\text { OPUS + } \\
\text { TR } \\
\text { TR }\end{array}$ & 2 años & \begin{tabular}{|l|} 
SCAN \\
SAPS \\
SANS \\
Life Chart \\
Schedule \\
GAF \\
\end{tabular} & \begin{tabular}{|l|} 
Línea Base \\
1,2 y 5 años de \\
seguimiento
\end{tabular} & $\begin{array}{l}2 \text { años: OPUS > TR } \\
\text { reducción de síntomas psi- } \\
\text { cóticos, abuso de sustancias, } \\
\text { medicación, uso de servicios } \\
\text { médicos, funcionamiento } \\
\text { global. } \\
5 \text { años: OPUS > TR } \\
\text { reducción del tiempo de } \\
\text { estancia hospitalario y uso de } \\
\text { viviendas tuteladas. }\end{array}$ & SI & $\begin{array}{l}\text { NO a los } 2 \\
\text { años } \\
\text { SI a los } 5 \\
\text { años }\end{array}$ \\
\hline $\begin{array}{l}\text { Gleeson et al } \\
(2009) 42\end{array}$ & $\begin{array}{l}82 \text { pacientes } \\
\text { en remisión } \\
\text { de síntomas } \\
\text { positivos tras } \\
\text { un primer } \\
\text { episodio } \\
\text { psicótico }\end{array}$ & $\begin{array}{l}\text { TCC } \\
\text { TR } \\
\end{array}$ & Durante 7 meses & $\begin{array}{l}\text { SCID MADRS } \\
\text { BPRS SANS } \\
\text { MARS WAIS } \\
\text { Premorbid Ad- } \\
\text { justment Scale } \\
\text { SOFAS } \\
\text { WHOQOL- } \\
\text { BREF ASSIST } \\
\text { AUDIT } \\
\end{array}$ & $\begin{array}{l}\text { Línea Base } \\
\text { Pos-trata- } \\
\text { miento } \\
\\
\\
\end{array}$ & $\begin{array}{l}\text { TCC }>\text { TR en reducción tasas } \\
\text { de recaída e incremento de } \\
\text { tiempo hasta la recaída } \\
\text { TCC=TR adherencia a la } \\
\text { medicación, mejora del } \\
\text { funcionamiento social y } \\
\text { calidad de vida } \\
\text { TCC<TR en reducción de } \\
\text { alogia medida por SANS } \\
\end{array}$ & SI & SI \\
\hline $\begin{array}{l}\text { McGorry et al } \\
\text { (2002)55 }\end{array}$ & $\begin{array}{l}59 \text { pacientes } \\
\text { con síntomas } \\
\text { subclínicos } \\
\text { en riesgo de } \\
\text { transición a la } \\
\text { psicosis }\end{array}$ & $\begin{array}{l}\text { IBN } \\
\text { IPE } \\
\end{array}$ & \begin{tabular}{|l|} 
Durante 6 meses, \\
variedad en las se- \\
siones atendiendo \\
a las necesidades \\
individuales de \\
los pacientes
\end{tabular} & \begin{tabular}{|l} 
BPRS \\
SANS \\
GAF
\end{tabular} & $\begin{array}{l}\text { Línea base } \\
\text { Post-trata- } \\
\text { miento } \\
12 \text { meses de } \\
\text { seguimiento }\end{array}$ & $\begin{array}{l}\text { IPE }>\text { IBN en post-tratamiento } \\
\text { IPE=IBN a los } 12 \text { meses de } \\
\text { seguimiento }\end{array}$ & SI & NO \\
\hline $\begin{array}{l}\text { Gumley et al } \\
\text { (2003)64 }\end{array}$ & $\begin{array}{l}144 \text { pacientes } \\
\text { en fase } \\
\text { prodrómica } \\
\text { o inicio de } \\
\text { recaída }\end{array}$ & $\begin{array}{l}\mathrm{TCC}+\mathrm{TR} \\
\mathrm{TR}\end{array}$ & \begin{tabular}{|l|}
5 sesiones iniciales \\
durante dos semanas \\
$2-3$ sesiones sema- \\
nales si aparecen \\
signos prodrómicos
\end{tabular} & $\begin{array}{l}\text { PANSS } \\
\text { BSI } \\
\text { PBIQ } \\
\text { SFS }\end{array}$ & \begin{tabular}{|l|} 
Línea base \\
Seguimiento a \\
las 12,26 y 56 \\
semanas
\end{tabular} & $\begin{array}{l}\text { TCC>TR mejora en } \\
\text { sintomatología psicótica y } \\
\text { general, funcionamiento } \\
\text { independiente y actividades } \\
\text { prosociales }\end{array}$ & SI & NO \\
\hline $\begin{array}{l}\text { Morrison } \\
\text { et al }(2004 \text {, } \\
2007) 60,63\end{array}$ & $\begin{array}{l}60 \text { pacientes } \\
\text { de alto riesgo } \\
\text { de transición } \\
\text { a la psicosis }\end{array}$ & \begin{tabular}{|l|} 
TCC + \\
supervisión \\
Supervi- \\
sión
\end{tabular} & $\begin{array}{l}\text { Un máximo } \\
\text { de } 26 \text { sesiones } \\
\text { durante } 6 \text { meses }\end{array}$ & \begin{tabular}{|l|} 
PANSS \\
Cuestionario \\
de Salud Gene- \\
ral de Goldberg \\
SCID \\
GAF
\end{tabular} & \begin{tabular}{|l|} 
Línea base \\
Post-trata- \\
miento \\
12 y 18 meses \\
de seguimiento
\end{tabular} & \begin{tabular}{|l|} 
TCC $>$ Supervisión, sinto- \\
matología de transición a \\
la psicosis, prescripción de \\
medicación antipsicótica y \\
probabilidad de presentar psi- \\
cosis, al postratamiento y a \\
los 12 meses de seguimiento \\
TCC $>$ Supervisión en pres- \\
cripción de medicación a los \\
18 meses de seguimiento \\
\end{tabular} & SI & NO \\
\hline $\begin{array}{l}\text { Addington et } \\
\text { al (2011)64 }\end{array}$ & $\begin{array}{l}51 \text { personas } \\
\text { en riesgo } \\
\text { de padecer } \\
\text { psicosis }\end{array}$ & \begin{tabular}{|l|} 
TCC \\
Terapia de \\
apoyo
\end{tabular} & \begin{tabular}{|l|}
20 sesiones \\
distribuidas a lo \\
largo de 6 meses
\end{tabular} & \begin{tabular}{|l} 
CDS \\
SAS \\
SIAS \\
SFS \\
WAI-SF
\end{tabular} & $\begin{array}{l}\text { Línea base } \\
6,12 \text { y } 18 \\
\text { meses }\end{array}$ & $\begin{array}{l}\text { TCC }>\text { TA en la rapidez de } \\
\text { mejora de los síntomas } \\
\text { prodrómicos a la psicosis } \\
\text { TCC }<\text { TA en la aparición de } \\
\text { brotes psicóticos }\end{array}$ & SI & NO \\
\hline
\end{tabular}


COPE= Cognitively-Oriented Psychotherapy for Early Psychosis; TCC= Terapia Cognitivo-Conductual; TR= Tratamiento Rutinario; $\mathrm{ACE}=$ Terapia cognitiva activa para la psicosis temprana; IBN= Intervención Basada en la Necesidad; IPE= Intervención Preventiva Específica; TA= Terapia de Apoyo; EMS= Explanatory Model Scale; SANS= Scale for the Assessment of Negative Symptoms; BPRS= Brief Psychiatric Rating Scale; SCLR-90= Symptoms Check List Revised; BDI= Beck Depression Inventory; QLS= Quality of Life Scale; SCAN= Schedule for Clinical Assessment in Neuropsychiatry; SAPS= Scale for Assessment of Psychotic Symptoms; $\mathrm{GAF}=$ Global Assessment of Functioning Scale; SCID= Structured Clinical Interview for DSM-IV; MADRS= Montgomery-Asberg depression rating Scale; MARS= Medication Adherence Rating Scale; WAIS= Wechsler Adults Intelligence Scale; SOFAS= Social and Occupational Functioning Assessment Scale; WHOQOL-BREF= World Health Organization Quality of Life Assessment-abbreviated version; ASSIST = Alcohol, Smoking and Substance Involvement Screening Test; AUDIT= Alcohol Use Disorders Identification Test; PANSS= Positive and Negative Syndrome Scale; BSI= Brief Symptom Inventory; PBIQ= Personal Beliefs about Illness Questionnaire; SFS= Social Funcioning Scale; CDS= Escala de Depresión de Calgary; SAS= Escala de Ansiedad Social; SIAS= Escala de Ansiedad ante la Interacción Social; WAI-SF= Inventario de Alianza Terapéutica.

aunque no en la preocupación por los delirios. Ambos grupos mostraron también una disminución en síntomas negativos y desorganización del pensamiento, pero sin llegar a tener diferencias entre ellos.

34 pacientes de esta misma cohorte fueron evaluados cinco años después (6) y aunque no se encontraron diferencias significativas en cuanto al grado de recaídas, los síntomas positivos o el insight entre ambos grupos, si se observó como la primera recaída tras la intervención se alargó mucho más en el grupo de TCC que en el TO, incrementándose también en el primer grupo la percepción de los pacientes sobre el control de su enfermedad con respecto a los otros.

La "terapia de cumplimiento" ("Compliance Therapy") ha sido la utilizada en otro estudio controlado (7). Dicha terapia consiste en una técnica que se conceptualiza al amparo de la entrevista motivacional (8) y cuyo objetivo principal es aumentar la adherencia al tratamiento farmacológico, fundamental en las fases agudas de las psicosis. Para su utilización con pacientes psicóticos se utiliza una postura terapéutica más activa, encaminada a la resolución de problemas y a incrementar el componente educacional. La "terapia de cumplimiento" (TC) implica una aproximación cognitiva a los síntomas psicóticos, siendo esta breve y pragmática, aplicable a pacientes en fase aguda. 47 pacientes fueron asignados aleatoriamente a dos grupos, a 25 se les aplicó la TC mientras que los otros 22 recibieron terapia de apoyo inespecífico (TAI). La TC consistió en un número de 4 a 6 sesiones de entre 20 y 60 minutos cada una, a razón de 2 sesiones semanales. El grupo control recibió el mismo número de sesiones durante el mismo tiempo, aplicadas por los mismos terapeutas. Las dos primeras sesiones de la TC consistían en una aproximación general del paciente a la historia de su enfermedad y a conceptualizar el problema, ayudado por el terapeuta; las dos siguientes sesiones consistían en una discusión más específica focalizada en los síntomas y en los efectos secundarios de la medicación, considerando los riesgos y beneficios del tratamiento farmacológico, explorando la ambivalencia del paciente a este respecto y subrayando el terapeuta las discrepancias entre las acciones y creencias del paciente; en las últimas sesiones se trataba el estigma de los fármacos, considerándolo como una libre 
ORIGINALES Y REVISIONES

elección del paciente para mejorar su calidad de vida. Se eligieron pacientes con psicosis funcional en fase aguda, independientemente del número de fase y del tipo de psicosis. Se evaluaron los resultados a los 3 y 6 meses y posteriormente en un seguimiento a 18 meses (9). Se observó una importante mejoría del grupo de TC con respecto al grupo control en las medidas de insight de la enfermedad y en la actitud con respecto al tratamiento farmacológico, así como una ligera mejoría en el funcionamiento social en general. Asimismo, los del grupo de TC tuvieron un mayor tiempo de permanencia general antes de una readmisión hospitalaria.

En la misma línea, O`Donell (10) y cols. realizaron un estudio aleatorio controlado y ciego con 56 sujetos diagnosticados de esquizofrenia con el objetivo de determinar si la TCC contribuía a una mejor adherencia y actitud hacia el tratamiento farmacológico, así como al insight. Las medidas de evaluación fueron: la sintomatología, medida por la PANSS (11), el insight, medido por la Schedule for Assessment of Insight (SAI) (12), el nivel general de funcionamiento, medido por la Global Assessment of Functioning Scale (GAF) (13), y la calidad de vida, medida por la Escala de Heinrich (QLS) (14). También se recogieron datos sobre el número de ingresos y la dosis de neurolépticos prescrita. Los sujetos se asignaron a dos condiciones experimentales: terapia de cumplimiento (TC) y grupo control. En este último los pacientes recibieron un asesoramiento no específico. En este estudio la TC no tuvo ninguna ventaja sobre la terapia no específica en ninguna de las variables estudiadas. Entre las limitaciones del estudio se encuentran un tamaño de muestra modesto y la utilización de informes de los pacientes, familiares y profesionales de la salud, en lugar de utilizar otro método más fiable de recogida de datos.

El estudio más grande y metodológicamente riguroso que investiga los efectos de la TCC en la reducción de síntomas, la prevención de recaídas y la velocidad del proceso de recuperación, de pacientes internos o ambulatorios con diagnóstico de esquizofrenia, trastorno esquizofreniforme, esquizoafectivo o delirante, fue llevado a cabo por Lewis (15) y cols. Se realizó una prueba controlada aleatoria, prospectiva y ciega a una muestra de 309 pacientes que cumplieron los requisitos mencionados en distintos centros de tratamiento. Las medidas de evaluación utilizadas fueron la escala PANSS (11) total, la subescala de síntomas positivos de dicha escala, y la escala PSYRATS. Se realizaron cinco evaluaciones: a los 14, 21 , 28 y 35 días, y una evaluación final de la fase aguda entre 42-70 días. Las condiciones de tratamiento fueron tres: TCC más tratamiento rutinario, terapia de apoyo más tratamiento rutinario y tratamiento rutinario sólo. La TCC fue desarrollada durante 5 semanas, con sesiones de refuerzo adicionales a las 2 semanas y una al mes durante los primeros tres meses. Dicha intervención estuvo manualizada y dividida en cuatro etapas. La primera etapa se destinó al contrato y a la valoración detallada del estado mental y a la educación sobre la naturaleza y el tratamiento de la psicosis. En la segunda etapa se generó una lista de problemas conjuntamente 
con el paciente, priorizados por el grado de angustia, viabilidad y riesgo clínico. La tercera y cuarta etapa se destinaron a la intervención (reestructuración cognitiva de los síntomas psicóticos positivos e identificación de factores precipitantes y de alivio de la sintomatología) y observación. La terapia de apoyo fue usada para controlar los elementos no específicos de una terapia psicológica y se llevó a cabo en las mismas condiciones que la TCC. Los resultados indicaron que los pacientes que recibieron TCC mostraron una tendencia hacia la mejora más rápida y semanal, alcanzando niveles estadísticamente significativos en la cuarta semana, aunque estas mejoras no persistieron en la evaluación final.

Posteriormente, el mismo grupo de autores $(16,17)$ publicó la segunda fase de este mismo estudio, consistente en la evaluación de la misma muestra, realizada a los 18 meses de seguimiento. Los resultados de entonces mostraron que el tratamiento psicológico resultó ser más beneficioso que el tratamiento rutinario en la reducción de la sintomatología medida por la escala PANSS; pero no así en la ideación delirante y las alucinaciones auditivas medidas por la escala PYSRATS. Sin embargo, no se encontró ninguna diferencia significativa entre la TCC y la terapia de apoyo. Tampoco se encontraron diferencias estadísticamente significativas en los índices de recaída o de rehospitalización en ninguna de las tres condiciones de tratamiento. Es necesario tener en cuenta el hecho de que se trataba de una muestra gravemente enferma, ya que un $38 \%$ de la misma se encontraban retenidos bajo la Ley de Salud Mental. En cuanto a las limitaciones de este estudio, es de destacar la existencia de una interacción entre centro y grupo de tratamiento. Otra limitación fue que no se estandarizó el tratamiento farmacológico, y que el tratamiento psicológico fue más breve de lo que se planeó en un principio.

Un análisis posterior (18) de este mismo estudio evaluó la eficacia de la TCC sobre la conducta suicida en comparación con las otras dos condiciones experimentales. No se encontraron diferencias significativas entre los tres grupos de tratamiento en ninguno de los periodos de tiempo evaluados, indicando que la TCC y la terapia de apoyo no reducen significativamente ni tampoco empeoran la conducta suicida en comparación con el tratamiento rutinario.

Otro subanálisis (19) de este estudio evalúa la eficacia de los distintos tratamientos en función de la edad del paciente, estableciendo dos puntos de corte: menor de 21 años y mayor de 21 años. Los autores encuentran que los pacientes menores de 21 años obtienen mayores beneficios de la terapia de apoyo que de la TCC mientras que los pacientes mayores de 21 años se benefician en mayor medida de la TCC que de la terapia de apoyo.

Una prueba aleatoria y controlada llevada a cabo por Startup (20) tuvo como objetivo evaluar la eficacia de la TCC para pacientes hospitalizados que sufrían episodios agudos de esquizofrenia y trastornos relacionados. La muestra estuvo compuesta por 90 pacientes que fueron asignados aleatoriamente a dos condiciones 
ORIGINALES Y REVISIONES

distintas de tratamiento: tratamiento rutinario o TCC más tratamiento rutinario. El tratamiento rutinario consistió en farmacoterapia y énfasis en los cuidados durante la hospitalización y cuidado de comunidad después del alta hospitalaria. La TCC siguió los objetivos y estrategias del manual publicado por Fowler y cols (21). Se trata de una intervención individualizada fundamentada en las necesidades de la TCC para los trastornos psicóticos y está basada en el empirismo de colaboración y la realización de formulaciones cognitivo-conductuales.

Las medidas de evaluación utilizadas fueron los síntomas positivos y negativos $\mathrm{y}$ el nivel de funcionamiento general, mediante las escalas SAPS (22) (Escala para la Evaluación de los Síntomas Positivos), SANS (23) (Escala para la Evaluación de los Síntomas Negativos), la BPRS-E (Expanded Brief Psychiatric Rating Scale) (24) y la GAF. Las medidas de evaluación se realizaron a los 6 y a los 12 meses del inicio del tratamiento. No se encontraron efectos significativos en la evaluación realizada a los 6 meses, a excepción de una disminución significativa de los síntomas de desorganización en la condición de TCC. Sin embargo, a los 12 meses, si se encuentra una mejoría significativa en la condición de TCC frente al tratamiento de rutina, para todas las variables medidas (excepto la desorganización).

Entre las limitaciones de este estudio está que los evaluadores no fueron ciegos a la condición de tratamiento, que no hubo control de efectos no específicos de la terapia psicológica y el número desigual de sesiones de TCC recibidas por cada paciente, que osciló entre 2 y 25.

Un año después, estos mismos autores (25) vuelven a evaluar la persistencia de los beneficios proporcionados por la TCC sobre los síntomas psicóticos y el funcionamiento social en la misma muestra de pacientes (a los 24 meses de seguimiento). Además, se evaluaron los costes comparativos de proporcionar TCC como un complemento al tratamiento rutinario frente al tratamiento rutinario en sí mismo. Las medidas utilizadas para las variables principales fueron la SAPS, la SANS, la SFS y el GAF. Para la evaluación económica de los servicios se utilizó el Service Utilization Schedule (SUS) (26) mediante la cual se identifica la cantidad de tiempo que el personal de salud mental emplea en actividades beneficiosas para el paciente. Debido a factores de mortandad experimental, la muestra quedó reducida al $73 \%$ de la original $(n=60)$. Los resultados obtenidos mostraron que todas las diferencias entre las condiciones de tratamiento fueron a favor de la TCC, si bien la significación estadística sólo se dio en los síntomas negativos y en el funcionamiento social, perdiéndose la ventaja previamente encontrada sobre los síntomas positivos. Por otro lado, no se encontraron diferencias significativas en los costes producidos por ambos grupos. Las limitaciones de este estudio fueron la carencia de ciego por parte de los evaluadores, la alta mortandad experimental y que la evaluación de los costes de los servicios se centró sólo en los 28 días precedentes a la realización de la entrevista. 


\section{Intervención temprana en psicosis}

Dentro de la intervención temprana en psicosis han sido llevados a cabo dos tipos de investigaciones: aquellas centradas en los primeros episodios psicóticos y aquellas que se dirigen hacia los signos tempranos o prodrómicos de la psicosis.

La COPE (Cognitively-Oriented Psychotherapy for Early-Psychosis) (27) es un tipo de terapia centrada en los primeros episodios psicóticos que se encuadra dentro del programa EPPIC (28) de detección e intervención temprana en psicosis llevado a cabo en Melbourne, Australia. La COPE es una terapia con origen en la metateoría del constructivismo pero que utiliza técnicas derivadas de la terapia cognitiva. A diferencia de los TCC que hemos visto hasta ahora, la COPE no tiene como objetivos per se los síntomas positivos de la psicosis, delirios y alucinaciones, basándose en el hecho real de que en los primeros episodios psicóticos un elevado porcentaje de pacientes responde de manera efectiva a los psicofármacos, mejorando sus síntomas positivos. La COPE tiene como objetivo ayudar al paciente en su ajuste después del episodio psicótico, cuando sus síntomas positivos han sido ya reducidos considerablemente. La psicoeducación y determinadas técnicas cognitivas se utilizaron para discutir el estigma y estereotipo que el propio paciente tenía de su enfermedad. Adicionalmente, se utilizaron también la técnicas cognitivas y conductuales necesarias para los problemas secundarios emergentes como la depresión y la ansiedad social. Para evaluar este tipo de intervención $(29,30)$ se eligieron 80 pacientes con su primer episodio psicótico, con diagnósticos muy heterogéneos (esquizofrenia, manía, depresión, psicosis sin especificar). 44 pacientes recibieron el programa integrado en la COPE, más el manejo de caso ("case management") y el tratamiento rutinario con psicofármacos y seguimiento (grupo COPE). A otros 21 pacientes que rechazaron la COPE se les aplicó sólo el manejo de caso y el tratamiento rutinario (grupo "rechazo"), y por último a un grupo control de 15 pacientes sólo se les aplicó el tratamiento rutinario (grupo control). La evaluación se llevó a cabo mediante diversos instrumentos:

- Parámetros psicológicos: la Escala del Modelo Explicativo (31) (Explanatory Model Scale), que mide la comprensión y creencias del paciente sobre su enfermedad, definiendo cinco dominios del modelo individual de enfermedad (etiología, tiempo y forma del desarrollo de los síntomas, patología, curso de la enfermedad y tratamiento); y la medida de I/SO (integración/retraimiento "sealing over"), que evalúa la adaptación individual a la enfermedad.

- Síntomas: se utilizó la BPRS, la SANS, la escala de depresión de Beck (BDI) (32), el índice general de síntomas (GSI) del SCL-90-R (33) y la escala de calidad de vida (QLS) de Heinrichs y Hanlon.

Los pacientes que recibieron la COPE tuvieron un número muy dispar de sesiones de tratamiento, por lo que se les dividió en dos subgrupos, aquellos con un 
ORIGINALES Y REVISIONES

número menor a 15 sesiones ( $\mathrm{n}=17 ; 3,82$ sesiones de media) y aquellos con $15 \mathrm{o}$ más sesiones ( $\mathrm{n}=27 ; 27,6$ sesiones de media). La COPE consistía fundamentalmente en cuatro fases, evaluación, enganche terapéutico, mejora en la adaptación a la psicosis e intervención sobre los problemas mórbidos secundarios, que muchas veces se superponían entre sí. La psicoeducación, el conocimiento de las deficiencias sociales por el propio paciente, el reconocimiento de sus estigmas y los problemas de motivación eran la base fundamental de la terapia. El resultado fue que hubo una significativa mejoría en los pacientes a los que se les aplicó la COPE con respecto al grupo control en cuanto a la adaptación individual a la enfermedad, medida por el I/SO, también en la comprensión y creencias del paciente sobre su enfermedad evaluadas por la escala del modelo explicativo, y en los síntomas negativos. También se obtuvo una mejoría significativa en la QLS, aunque esta fue tan solo evaluada después del tratamiento y no antes. Un aspecto reseñable es que el grupo "rechazo" mejoró significativamente en las puntuaciones del BDI con respecto al grupo COPE al final del tratamiento.

Tras este estudio preliminar, este mismo grupo de autores realiza un estudio controlado (34) con 91 pacientes, integrados dentro del programa EPPIC, y que sufrían un primer episodio psicótico, utilizando condiciones y medidas similares a las señaladas anteriormente. De los 91 pacientes disponibles, 45 fueron seleccionados al grupo COPE (tratamiento COPE más cuidado rutinario EPPIC) y los 46 restantes constituyeron el grupo no-COPE (cuidado rutinario EPPIC). De los 45 pacientes que inicialmente conformaron el grupo COPE, 12 rehusaron recibir este tratamiento, quedando reducido a 33 pacientes. En este caso, los autores no encuentran una mejoría significativa del grupo de tratamiento COPE comparado con el no-COPE, en ninguna de las medidas para ninguno de los momentos temporales medidos (seguimiento tras 6 y 12 meses de finalizar el tratamiento). Sin embargo, este estudio adolece de varias limitaciones significativas. Se trata de un estudio no aleatorizado, donde es posible que los pacientes recibieran un número menor de sesiones de las suficientes; la media de sesiones recibidas es 15,4 (mediana 10) mientras que en otros estudios similares se encuentran en torno a una media de sesiones de 18-21. Además, pese que el tratamiento COPE estaba manualizado, no estaba suficientemente protocolarizado, lo que pudo llevar a que algunos pacientes recibieran un tratamiento incompleto.

Otro estudio desarrollado en el EPPIC (35) seleccionó a sesenta y dos personas que se encontraban el la fase aguda de su primer episodio psicótico y que dieron su consentimiento por escrito para participar en el estudio. Los participantes completaron una entrevista inicial para determinar el diagnóstico, la corriente, la medicación, la sintomatología y el funcionamiento de la vida. Otras evaluaciones se llevaron a cabo a las 6 semanas, 12 semanas y 15 meses después de la línea base (es decir, 1 año después del tratamiento). Las siguientes variables demográficas y 
relacionadas con la enfermedad fueron evaluadas: sexo, edad, educación, estado civil, edad de aparición de la enfermedad, duración de la psicosis no tratada, hospitalizaciones, dosis de neurolépticos, y la recepción de terapia electroconvulsiva (TEC). La psicosis primaria o cualquier otra categoría diagnóstica en el eje I se evaluó mediante la SCID (36). Para la valoración de los síntomas psicóticos positivos se utilizó la subescala de síntomas psicóticos (37) de la Escala Breve de Valoración Psiquiátrica (BPRS) (38). La SANS (23) se utilizó para evaluar los síntomas psicóticos negativos; y la SOFAS (39) para la evaluación del funcionamiento Ocupacional y Social. Tras la evaluación inicial, los participantes fueron asignados aleatoriamente a uno de las dos condiciones de tratamiento protocolizado: una condición de TCC llamada "Terapia cognitiva activa para la psicosis temprana" ("ACE") (40) o una condición de control llamada "Entablamento de amistad" (41).

Los resultados mostraron una tendencia notable de la ACE para mejorar los resultados (en términos de desaparición de los síntomas negativos y mayor funcionamiento en particular) a mitad de tratamiento y, en menor medida, al final del tratamiento. Sin embargo, respecto a la comparación entre tratamientos, ambos grupos mejoraron significativamente con el tiempo. ACE superó significativamente a Entablamento de Amistad mediante la mejora del funcionamiento a la mitad del tratamiento, pero no mejoró los síntomas positivos o negativos. Más allá de la evaluación a mitad de tratamiento, no se encontraron diferencias significativas en ninguna medida de resultado y en ingresos hospitalarios durante el seguimiento, lo que confirma que existe alguna evidencia preliminar de que ACE promueve una mejor recuperación temprana del funcionamiento aunque estos resultados deberían ser replicados en otros centros de investigación con muestras más amplias.

Este mismo grupo de autores (42) se evalúa la eficacia de la TCC en comparación con el tratamiento de rutina en la prevención de recaídas en pacientes tras un primer episodio psicótico. 82 pacientes en remisión de síntomas positivos tras un primer episodio de psicosis fueron incluidos en el estudio. Los pacientes fueron aleatorizados a las dos condiciones posibles de tratamiento: TCC o tratamiento de rutina (43). La TCC fue llevada a cabo siguiendo las fases manualizadas por el programa EPPIC (26) y se dirigió a dos vertientes: individual y familiar. Las evaluaciones fueron realizadas por evaluadores ciegos y se llevaron a cabo las siguientes medidas: SCID $(36,44)$ Escala de depresión de Montgomery-Asberg (MADRS) (45), escala BPRS (38), escala SANS (23), Escala de Evaluación de la adherencia a la medicación (MARS) (46), Escala Wechsler de Inteligencia para adultos (47), Premorbid Adjusment Scale (48), the Social and Occupational Functioning Assessment Scale (49), y una versión australiana de la World Heatlh Organization Quality of Life Assessment-abbreviated version (WHOQOL-BREF) (50). Para evaluar el consumo de sustancias utilizaron: WHO Alcohol, Smoking and Substance Involvement Screening test (51) y the Alcohol Use Disorders Iden- 
ORIGINALES Y REVISIONES

tification test (52). También fueron recogidos el número de ingresos hospitalarios y la duración de estos. El análisis de resultados fue realizado teniendo en cuenta la línea base (inicio del tratamiento) y un periodo de seguimiento de 7 meses (finalización del tratamiento). Encontraron que la TCC era significativamente superior que el tratamiento rutinario en la reducción de las tasas de recaída así como en la prolongación del tiempo hasta la recaída. No encontraron diferencias entre los dos grupos en cuanto a la adherencia a la medicación, mejora del funcionamiento social o de la calidad de vida, mostrando los dos grupos una mejoría significativa en la comparación inicio del tratamiento y seguimiento a los 7 meses. Asimismo, el grupo de TCC mostró en el seguimiento una mayor puntuación en la subescala SANS referida a la alogia, comparada con el tratamiento de rutina.

Para estudiar el mantenimiento de los efectos a largo plazo de los programas de intervención temprana, se diseñó un estudio (53) que evaluó los efectos diferenciales de un programa intensivo de intervención temprana respecto al tratamiento de rutina. Un total de 547 pacientes con un primer episodio de diagnóstico en el espectro de la esquizofrenia fueron incluidos. Se distribuyeron aleatoriamente a la condición de tratamiento intensivo (OPUS) o tratamiento rutinario. El tratamiento intensivo tuvo una duración de dos años y estuvo centrado en los siguientes elementos: tratamiento asertivo comunitario, terapia familiar y entrenamiento en habilidades sociales, teniendo como eje central el ofrecer un tratamiento individualizado e intensivo para cada paciente. El tratamiento farmacológico se llevó a cabo siguiendo las recomendaciones de guías clínicas para primeros episodios psicóticos, a dosis bajas y con antipsicóticos de segunda generación. Una vez transcurridos los dos años de intervención, los pacientes del grupo intensivo pasaban de forma gradual a recibir el tratamiento rutinario. Se realizaron evaluaciones al inicio del tratamiento y a los 1,2 y 5 años tras el inicio del tratamiento, tomando como medidas comparativas de evaluación las registradas a los 2 y 5 años. Como medidas de evaluación tomaron los síntomas psicóticos negativos y positivos (medidos a través de las escalas SANS (23) y SAPS (22) y el funcionamiento global (medido a través de la escala GAF). También hicieron evaluaciones secundarias del abuso de sustancias, las dosis de medicación y el uso de servicios médicos, síntomas depresivos, conductas suicidas, y situación laboral y de vivienda. Este grupo de autores encuentra que el tratamiento intensivo resulta significativamente más eficaz que el tratamiento de rutina en la evaluación realizada a los 2 años. Así, se mostró una mayor reducción de síntomas psicóticos negativos y positivos y abuso de sustancias, los pacientes tomaron dosis significativamente inferiores de antipsicóticos, el tiempo de estancia media hospitalaria fue inferior, y el funcionamiento global superior. Sin embargo, los beneficios obtenidos en la evaluación a los 2 años no se mantuvieron a los 5 años en casi todas las variables evaluadas. Así, los dos grupos no difieren en la evaluación a los 5 años en la reducción de síntomas psicóticos negativos y positivos, funcionamiento glo- 
bal, abuso de sustancias, depresión y conducta suicida. Si encontraron diferencias en cuanto al tiempo de estancia de las hospitalizaciones y al uso de hogares protegidos, a favor del grupo de intervención intensiva, mostrando estos una mayor adaptación a la vida fuera de las instituciones.

Con el objetivo de comprobar si los resultados obtenidos a los 2 años de seguimiento se pudieran extender y mantener en el tiempo incrementando la duración de la intervención intensiva en el periodo crítico, este mismo grupo de autores ha diseñado un estudio (54) en el que se comparan los efectos del tratamiento intensivo (OPUS) llevado a cabo durante 2 años con el mantenido durante 5 años. Habrá que esperar a la publicación de estos resultados para evaluar si el no mantenimiento de los efectos a largo plazo se debe a la necesidad de un tratamiento intensivo más prolongado o a otros factores.

Con respecto a los grupos de investigación centrados en el concepto de pródromo y que tratan de identificar e intervenir sobre éstos con el objetivo de prevenir el comienzo de la enfermedad, uno de los primeros estudios realizados respecto a esta temática fue el llevado a cabo por McGorry (55). Se trata de una prueba aleatoria controlada de intervenciones designadas a reducir el riesgo de progresión al primer episodio de psicosis en una muestra de 59 pacientes con síntomas subclínicos. Para ello se comparó la eficacia de una Intervención Basada en la Necesidad (IBN) frente a una Intervención Preventiva Específica (IPE). La IBN comprende psicoterapia de apoyo principalmente enfocada en cuestiones tales como las relaciones familiares, sociales y laborales. Los pacientes en este grupo no recibieron medicación antipsicótica, sino que recibían antidepresivos si presentaban depresión moderada o severa y benzodiacepinas si sufrían de insomnio. Por otro lado, la IPE comprende todos los elementos de la terapia anterior y dos componentes adicionales: administración de 1 o $2 \mathrm{mg}$. de Risperidona diariamente durante 6 meses y una TCC manualizada por los mismos autores, cuyos objetivos generales fueron el entendimiento de los síntomas experimentados, aprendizaje de estrategias de control de dichos síntomas y reducción del estrés asociado. Como medidas de evaluación se utilizaron la BPRS, la Escala de Evaluación de Síntomas Negativos, la escala de Hamilton para la Depresión y Ansiedad (56), la Young Manía Scale (57), la Schedule for Clinical Assessment in Neuropsychiatry (58), Comprehensive Assessment of at-risk Mental Status (59), la Escala de Calidad de Vida y la Escala de Evaluación del Funcionamiento Global. Las evaluaciones se realizaron al finalizar el tratamiento ( 6 meses) y a los 6 meses de finalizarlo (12 meses tras el inicio). Los resultados indican que al final del tratamiento hubo diferencias significativas entre los grupos con respecto al porcentaje de transición a la psicosis, observándose ventajas para el grupo IPE. Sin embargo, después de 6 meses de seguimiento estas diferencias no se mantuvieron significativas, aunque en los pacientes del grupo IPE la protección contra la progresión a la psicosis se extendió durante 6 meses después de retirar la administración de Risperidona. 
ORIGINALES Y REVISIONES

En un estudio llevado a cabo por Morrison (60), se evaluó si la terapia cognitiva reducía el porcentaje de transición a la psicosis y la prescripción de medicación antipsicótica, así como la severidad de los síntomas subclínicos en personas con alto riesgo de padecer psicosis. Se seleccionó una muestra de 60 pacientes que cumplían el criterio de alto riesgo, operativizado por la presencia de síntomas transitorios psicóticos o síntomas psicóticos atenuados (medidos a través de la PANSS), la presencia de un estado de alto riesgo mental (Cuestionario de salud general de Goldberg (61) ) y/o un deterioro reciente (GAF), el historial de familia (paciente de primer grado con una historia de cualquier trastorno psicótico), o un diagnóstico de trastorno de personalidad esquizotípico (SCID) $(28,46)$. Para medir la transición a la psicosis se utilizaron las subescalas del PANSS, la frecuencia y duración de los síntomas, la prescripción de medicación antipsicótica o la realización de un probable diagnóstico DSM-IV por parte de un médico independiente o ciego. Se trata de un estudio aleatorio y controlado, cuya asignación a las condiciones de tratamiento fue estratificada mediante las variables riesgo sexual y genético. Hubo dos condiciones de tratamiento: supervisión y TCC más supervisión. La TCC fue desarrollada bajo los principios del modelo de Beck (62), cuyo componente central se fundamentó en la normalización de las interpretaciones del paciente y la búsqueda de explicaciones alternativas, descatastrofizando sus temores y reevaluación de sus interpretaciones mediante el uso de experimentos conductuales. Se encontró una reducción del $96 \%$ en la sintomatología de transición a la psicosis de la TCC frente al grupo control, un $94 \%$ de prescripción de medicación antipsicótica y un $96 \%$ de probabilidad de presentar un trastorno psicótico. Por el contrario, no se encontró ninguna evidencia de que la TCC mejore el funcionamiento global o la angustia, aunque hay que tener en cuenta que para estas medidas hubo una gran cantidad de datos perdidos. En cuanto a las limitaciones, el estudio contaba con un tamaño de muestra pequeño, no se pudo mantener el enmascaramiento de los evaluadores, el método de aleatorización causó tamaños de grupo desiguales y no se controlaron efectos no específicos de la relación con el terapeuta.

El mismo grupo de autores publica un análisis posterior (63) del mismo estudio en el que analizan los resultados obtenidos a los 3 años de seguimiento. En este caso encuentran que los pacientes que recibieron TCC necesitaron una menor dosis de medicación e iniciaron el tratamiento farmacológico más tardíamente. No encuentran diferencias significativas en cuanto a la reducción de la probabilidad de transición a la psicosis ni en el diagnóstico DSM-IV.

En otro estudio reciente realizado por el Centro para la Adicción y Salud Mental de Toronto (Canadá) sobre personas en estadios prepsicóticos (64) se comparó la eficacia de la TCC frente a la terapia de apoyo en una muestra de 51 personas con alto riesgo de desarrollar psicosis. Los siguientes instrumentos de evaluación fueron administrados a los 6,12 y 18 meses de seguimiento: Escala de 
Depresión de Calgary (CDS) (65), Escala de Ansiedad Social (SAS) y Escala de Ansiedad ante la interacción Social (SIAS) (66); Escala de Funcionamiento Social (SFS) (67) y la forma breve del Inventario de Alianza terapéutica (WAI-SF) (68). Los resultados mostraron que la aparición de los brotes psicóticos sólo se dio en el grupo que estaba recibiendo Terapia de apoyo, aunque la diferencia no fue significativa. Ambos grupos mejoraron los síntomas positivos prodrómicos, la depresión y la ansiedad social, aunque ninguno mejoró el funcionamiento social y los síntomas negativos. Aunque no hubo diferencias en ambos grupos, se demostró que en la TCC la mejoría de los síntomas positivos prodrómicos fue más rápida.

Otro estudio cuyo objetivo fue evaluar la eficacia de la TCC llevada a cabo como una estrategia de intervención temprana para prevenir recaídas fue el llevado a cabo por Gumley (69). Consistió en una prueba aleatoria controlada de TCC aplicada durante el pródromo o signos tempranos de recaída en la esquizofrenia. 144 pacientes con esquizofrenia o un trastorno relacionado fueron asignados aleatoriamente a dos condiciones experimentales: tratamiento rutinario y TCC más tratamiento rutinario. Los componentes principales del tratamiento rutinario fueron: la medicación en curso, la revisión psiquiátrica regular y el seguimiento regular por un trabajador de referencia, por lo general, personal de enfermería de equipos de salud mental comunitarios. La TCC, llevada a cabo por un psicólogo clínico, fue dividida en dos fases: una fase de contrato desarrollada entre el inicio y la semana 12 de tratamiento, y una fase intensiva (2-3 sesiones por semana) realizada ante la aparición de los signos tempranos de recaída. Posteriormente se hizo un seguimiento durante 12 meses, con evaluaciones en la línea base y a las 12, 26 y 56 semanas. Los síntomas positivos y negativos fueron medidos usando la PANSS, el estrés psicológico se midió usando el Brief Symptom Inventory (BSI) (70), las apreciaciones negativas de psicosis fueron evaluadas usando el Personal Beliefs about Illness Questionnaire (PBIQ) (71), la escala de autoestima de Rosenberg fue seleccionada como una medida de las apreciaciones negativas de uno mismo, la Escala de funcionamiento social (SFS) (67) fue usada para evaluar el papel social y el funcionamiento conductual. Se encontró que a los 12 meses las tasas de recaída e ingresos hospitalarios eran más bajas en el grupo TCC que en el grupo control, sin embargo, los resultados de los ingresos hospitalarios no fueron significativos. También se alcanzaron mejoras significativas en los síntomas negativos, positivos, sintomatología general, funcionamiento independiente y actividades prosociales. Aunque los resultados son alentadores, hay que tener en cuenta que carecía de evaluadores ciegos, lo que pudo ser una fuente de sesgos en los resultados obtenidos. 
ORIGINALES Y REVISIONES

\section{Conclusión}

Desde hace años, la intervención psicoterapéutica en psicosis crónicas está instaurada en los sistemas sanitarios españoles, mostrando los beneficios que esta intervención proporciona en la evolución y curso de esta enfermedad. Este hecho, lejos de quedar estático, ha ido extendiéndose a otras esferas de la psicosis, entre las que se sitúan las psicosis agudas, anteriormente relegadas únicamente al tratamiento farmacológico. El hecho de que las intervenciones psicológicas cognitivoconductuales se hayan mostrado eficaces en la reducción de síntomas refractarios así como en otra de la sintomatología asociada a la psicosis, ha contribuido a la extensión del modelo hacia áreas en las que anteriormente se mostraba una mayor reticencia en su abordaje psicológico.

La terapia cognitivo-conductual, combinada con el tratamiento farmacológico, se va abriendo paso como una herramienta eficaz en el abordaje de la psicosis agudas, mostrando un mayor beneficio en su uso combinado que el tratamiento farmacológico únicamente. Los resultados mostrados hasta el momento respecto a la eficacia de la terapia cognitivo-conductual en el contexto de una fase aguda permiten un cierto grado de optimismo en su consideración como tratamiento complementario al psicofarmacológico, mostrando unos mejores resultados en la prevención de recaídas, la adhesión al tratamiento farmacológico y la sintomatología negativa.

Aunque el tratamiento farmacológico sigue siendo un elemento esencial en el proceso terapéutico de la psicosis, el tratamiento psicológico supone un componente imprescindible para una recuperación integral y satisfactoria de los pacientes psicóticos.

Por otro lado y, en los últimos años, la intervención en psicosis ha centrado sus esfuerzos en la intervención precoz, como una estrategia que permita mejorar el curso de la enfermedad. Los análisis mostrados hasta la fecha indican resultados prometedores, lo que alienta a continuar en esta línea.

$\mathrm{El}$ interés por la intervención precoz ha sufrido un gran incremento en los últimos tiempos, lo que ha generado un importante número de publicaciones así como la creación de sociedades dedicadas al trabajo específico en intervención temprana. Asimismo, los estamentos políticos no han permanecido al margen, realizando reformas asistenciales que permitan el progreso de este nuevo paradigma.

La situación actual permite observar una realidad muy diferente a la que ocurría no mucho tiempo atrás. Los avances en los tratamientos farmacológicos (cada vez más eficaces y con menores efectos secundarios) y la evidencia de la validez de las intervenciones psicológicas han permitido una notable evolución en la asistencia en la salud mental. De este modo, las psicosis ya no son consideradas enfermedades severas y degenerativas para las que no existe un tratamiento eficaz sino 
que, por el contrario, existe una ola de optimismo en el tratamiento de la psicosis, teniendo como colofón final la intervención temprana.

\section{BIBLIOGRAFÍA:}

(1) McGorry PD, Killackey E, Yung A. Early intervention in psychosis: concepts, evidence and future directions. World Psychiatry 2008; 7: 148-156.

(2) Haddock G, Morrison A, Hopkins R, Lewis S, Tarrier N. Individual cognitive-behavioural interventions in early psychosis. Br J Psychiatry 1998; 172 Suppl 33: 101-106.

(3) Drury V, Birchwood M, Cochrane R, McMillan F. Cognitive therapy and recovery from acute psychosis: a controlled trial. I: Impact on psychotic symptoms. Br J Psychiatry 1996a; 169: 593-601.

(4) Drury V, Birchwood M, Cochrane R, McMillan F. Cognitive therapy and recovery from acute psychosis: a controlled trial. II: Impact on recovery time. Br J Psychiatry 1996b; 169: 602-607.

(5) Liberman, R. P., Wallace, C. J., Falloon, I. R. H. y Vaughn, E. Psychiatric Assessment Scale (PAS). Comprehensive Psychiatry 1981; 22 (6).

(6) Drury V, Birchwood M, Cochrane R. Cognitive therapy and recovery from acute psychosis: a controlled trial. Five-year follow-up. Br J Psychiatry 2000; 177: 8-14.

(7) Kemp R, Hayward P, Applewhaite G, Everitt B, David A. Compliance therapy in psychotic patients: randomised controlled trial. BMJ 1996; 312: 345-349

(8) Rollnick S, Heather N, Bell A. Negotiating behaviour change in medical settings: The development of brief motivational interviewing. J Ment Health 1992; 1: 25-37.

(9) Kemp R, Kirov G, Everitt B, Hayward P, David A. Randomised controlled trial of compliance therapy. 18-month follow-up. Br J Psychiatry 1998; 172: 413-419.

(10) O`Donnell C, Donohoe G, Sharkey L, Owens N Migone M, Harries R, Kinsella A, et al. Compliance therapy: a randomised controlled trial in schizophrenia. BMJ 2003; 327 (7419):834.

(11) Kay S, Fiszbein A, Opler L. The Positive and Negative Syndrome Scale (PANSS). Schizophr Bull 1987; 13: 261-273

(12) David AS. Insight and psychosis. Br J Psychiatry 1990; 156: 798-808.

(13) American Psychiatric Association. Diagnostic and Stadistical Manual of Mental Disorders, Fourth Edition. Washington, DC: American Psychiatric Association; 1994.

(14) Heinrichs DW, Hanlon TE, Carpenter WT Jr. The quality of Life Scale: and instrument for rating the schizophrenic deficit syndrome. Schizophr Bull 1984; 10: 338-398.

(15) Lewis S, Tarrier N, Haddock G, Bentall R, Kinderman P, Kingdon D, et al. Randomised controlled trial of cognitive behavioural therapy in early schizophrenia: acute-phase outcomes. Br J Psychiatry 2002 Sept; Suppl 43: 91-97.

(16) Tarrier N, Lewis S, Haddock G, Bentall R, Kinderman P, Kingdon D, et al. Cognitive behavioural therapy in first episode and early schizophrenia. Br J Psychiatry 2004; 184: 231-239.

(17) Tarrier N, Lewis S, Haddock G, Bentall R, Drake R, et al. Cognitive behavioural therapy in first- episode and early schizophrenia 18-month follow-up of a randomised controlled trial. Br J Psychiatry 2004; 184: 231-239.

(18) Tarrier N, Haddock G, Lewis S, Drake R, Gregg L. Suicide behaviour over 18 months in recent onset schizophrenic patients: The effects of CBT. Schizophr Res 2006; 83: 15-27. 
(19) Haddock G, Lewis S, Bentall R, Dunn G, Drake R, Tarrier N. Influence of age on outcome of psychological treatments in first-episode psychosis. Br J Psychiatric 2006; 188: 250-254.

(20) Startup M, Jackson MC, Bendix, S. North Wales randomised controlled trial of cognitive behaviour therapy for acute schizophrenia spectrum disorders: outcomes at 6 and 12 months. Psychol Med 2004; 34: 413-422

(21) Fowler D, Garety P, Kuipers E. Cognitive Behaviour Therapy for Psychosis: Theory and Practice. Chichester: Wiley, 1995.

(22) Andreasen NC. Scale for the Assessment of Positive Symptoms (SAPS). University of Iowa: Iowa City; 1984.

(23) Andreasen NC (1984). Scale for the Assessment of Negative Symptoms (SANS). University of Iowa: Iowa.

(24) Lukoff D, Nuechterlein KH, Ventura J. Symptom monitoring in the rehabilitation of schizophrenic patients. Schizophr Bull 1986; 12:578-602.

(25) Startup M, Jackson MC, Evans KE, Bendix S. North Wales randomised controlled trial of cognitive behaviour therapy for acute schizophrenia spectrum disorders: two year follow-up and economic evaluation. Psychol Med 2005; 35: 1307-1316.

(26) Prendergast LA, Griffith GL, Evans KE, Carter MF, Crosby C. A study of continuing care clients of community mental health teams. Unpublished report. Health Services Research Unit (1999), University of Wales, Bangor, UK.

(27) Henry L, Edwars J, Jackson HJ, Hulbert C, McGorry PD. Cognitively-oriented psychotherapy for early psychosis (COPE): a Practitioner's Manual, EPPIC: the Early Psychosis Prevention and Intervention Center, 2002

(28) McGorry P, Edwards J, Mihalopoulis C, Harrigan SM, Jackson HJ. EPPIC: An envolving system of early detection and optimal management. Schizophr Bull 1996, 22: 305-326.

(29) Jackson H, McGorry P, Edwards J, Hulbert C, Henry L, Francey S et al. Cognitively-oriented psychotherapy for early psychosis (COPE). Preliminary results. Br J Psychiatry 1998; 172 Suppl 33: 93-100.

(30) Jackson H, McGorry P, Henry L, Edwards J, Hulbert C, Harrigan S, et al. Cognitivelyoriented psychotherapy for early psychosis (COPE): A one-year follow-up. Br J Clinic Psychol 2001; 40: 57-70.

(31) Kleinman A. Patients and Healers in the Context of Culture. An Exploration of the Borderline between Anthropology, Medicine and Psychiatry. University of California Press Ltd: Berkeley, California; 1980.

(32) Beck AT, Ward CH, Mendelson M, Mock J, Erbaugh J. An inventory for measuring depression. Arch Gen Psychiatry 1961; 4:561-571.

(33) Derogatis LR. The SCL-90-R. Baltimore, MD: Clinical Psychometric Research, 1975.

(34) Jackson H, McGorry P, Henry L, Edwards J, Hulbert C, Henry L, et al. A controlled trial of cognitively oriented psychotherapy for early psychosis (COPE) with four-year follow-up readmission data. Psychol Med 2005; 35: 1295-1306.

(35) Jackson HJ, McGorry PD, Killackey E, Bendall S, Allott K, Dudgeon P, et al. Acute-phase and 1-year follow-up results of a randomized controlled trial of CBT versus Befriending for first-episode psychosis: the ACE project Psychol Med 2008; 38(5):725-35.

(36) First MB, Spitzer RL, Gibbon M, Williams JBW. Structured Clinical Interview for DSMIV-TR Axis I Disorders-Research Version, Patient Edition (SCID-I/P). Biometrics Research Department, 
New York State Psychiatric Institute: New York: 2001.

(37) Harrigan SM, McGorry PD, Krestev H. Does treatment delay in first-episode psychosis really matter? Psychol Med 2003; 33, 97-110.

(38) Ventura J, Lukoff D, Nuechterlein KH, Liberman RP, Green MF, Shaner A. Brief Psychiatric Rating Scale (BPRS) Expanded Version (4.0). Scales, Anchor Points, and Administration Manual. UCLA Department of Psychiatry and Behavioral Sciences: West Los Angeles: 1993.

(39) APA. Diagnostic and Statistical Manual of Mental Disorders, 4th edn. American Psychiatric Association : Washington, DC: 1994.

(40) Bendall S, Killackey E, Marois MJ, Jackson H. ACE Manual (Active Cognitive Therapy for Early Psychosis). ORYGEN Research Centre and Department of Psychology, University of Melbourne: Melbourne; 2005.

(41) Bendall S, Killackey E, Jackson H, Gleeson J. Befriending Manual. ORYGEN Research Centre and Department of Psychology, University of Melbourne: Melbourne; 2003.

(42) Glesson JFM, Cotton SM, Alvarez-Jimenez M, Wade D, Gee D, Crisp K, et al. A randomized controlled trial of relapse prevention therapy for first-episode psychosis patients. J Clin Psychiatry 2009; 70(4): 477-486.

(43) Early Psychosis Prevention and Intervention Centre. Case management in early psychosis: a handbook. EPPIC: Parkville, Victoria, Australia: 2001.

(44) First MB, Spitzer RL, Williams JBW, Gibbon M. Structured Clinical Interview for DSM-IV Axis II Personality Disorders (SCID-II). User's Guide and Interview. Washington, DC: American Psychiatric Press: 1997.

(45) Montgomery SM. Depressive symptoms in acute schizophrenia. Prog Neuropsychopharmacol, 1979; 3:429-433.

(46) Thompson K, Kulkarni J, Sergejew AA. Reliability and validity of a new Medication Adherence Rating Scale (MARS) for the psychoses. Schizophr Res 2000; 42: 241-247.

(47) Ginsberg JP. Wechsler Test of Adult Reading. Applied Neuropsychol 2003; 10:182-184.

(48) Cannon-Spoor HE, Potkin SG, Wyatt RJ. Measurement of premorbid adjustment in chronic schizophrenia. Schizophr Bull 1982; 8: 470-484.

(49) Goldman HH, Skodol AE, Lave TR. Revising Axis-V for DSM-IV: a review of measures of social functioning. Am J Psychiatry 1992; 149: 1148-1156.

(50) Development of the World health Organization WHOQOL-BREF quality of life assessment. The WHOQOL Group. Psychol Med 1998; 28(3): 551-558.

(51) Ali R, Awwad E, Babor T, Bradley F, Butau T, Farrell M, et al. The Alcohol, Smoking and Substance Involvement Screening Test (ASSIST): development, reliability and feasibility. Addiction 2002; 97: 1183-1194.

(52) Saunders JB, Aasland OG, Babor TF, de la Fuente JR, Grant M. Development of the Alcohol Use Disorders Identification Test (AUDIT): WHO Collaborative Project on Early Detection on Persons with Harmful Alcohol Consumption. Addiction 1993; 88(6): 791-804.

(53) Bertelsen M, Jeppesen P, Petersen L, Thorup A, Ohlenschlager J, Quach P, et al. Five-year follow-up of a randomized multicenter trial of intensive early intervention vs standard treatment for patients with a first episode of psychotic illness. Arch Gen Psychiatry 2008, 65(7):762-71.

(54) Melau M, Jeppesen P, Thorup A, Bertelsen M, Petersen L, Gluud C, et al. The effect of five years versus two years of specialised assertive intervention for first episode psicosis- OPUS II: study pro- 
tocol for a randomized controlled trial. Trials 2011, 12:72.

(55) McGorry P, Yung A, Phillips L, Yuen, H, Francey S, Cosgrave E, et al. Randomised controlled trial of interventions designed to reduce the risk of progression to first-episode psychosis in a clinical sample with subthreshold symptoms. Arch Gen Psychiatry 2002; 59: 921-928.

(56) Hamilton M. The Assessment of Anxiety States by Rating. Br J Psychiatry 1959; 32: 50-55.

(57) Young RC, Biggs JT, Ziegler VE, Meyer DA. A Rating Scale for Mania: reliability, validity and sensitivity. Br J Psychiatry 1978; 133: 429-435.

(58) Wing JK, Babor T, Brugha T, Burke J. SCAN: Schedules for clinical assessment in neuropsychiatry. Arch Gen Psychiatry 1990; 47: 589-593.

(59) Yung AR. The Comprehensive Assessment of At-risk Mental States (CAARMS). Melbourne, Australia: University of Melbourne; 2000.

(60) Morrison AP, French P, Walford L, Lewis SW, Kilcommons A, Green J, et al. Cognitive therapy for the prevention of psychosis in people at ultra-high-risk: Randomised controlled trial. Br J Psychiatry 2004; 185: 291-297.

(61) Goldberg DP, Hillier VF. A scale version of the general health questionnaire. Psychol Med 1979; 9: 139-145.

(62) Beck AT. Cognitive Therapy and the Emotional Disorder. New York: International Universities Press; 1976.

(63) Morrison AP, French P, Parker S, Roberts M, Stevens H, Bentall RP et al. Three-Year Follow-up of a Randomized controlled trial og cognitive therapy for the prevention of psychosis in people at ultrahigh risk. Schizophr Bull 2007; 33(3): 682-687.

(64) Addington J, Epstein I, Liu L, French P, Boydell KM, Zypursky RB. A randomized controlled trial of CBT for individuals at clinical high risk of psychosis. Schizopr Res 2011; 125: 54-61.

(65) Addington D, Addington J, Maticka-Tyndale E. Assessing depression in schizophrenia: the Calgary Depression Scale. Br J Psychiatry 1993; 163, 39-44.

(66) Olivares J, Garcia-Lopez LJ, Hidalgo MD. The Social Phobia Scale and the Social Interaction Anxiety Scale: factor structure and reliability in a Spanish speaking population. J Psychoeduc Assess $2001 ; 19,69-80$.

(67) Birchwood M, Smith J, Cochrane R, Wetton C, Copestake C. The Social Functioning Scale: the development and validation of a new scale of social adjustment for use in family intervention programmes with schizophrenic patients. Br J Psychiatry 1990; 157: 853-859.

(68) Tracey T, Kokotovic A. Factor Structure of the Working Alliance Inventory. Psychological Assessment 1989; 1: 207-210.

(69) Gumley A, O`Grady M, McNay L, Reilly J, Power K, Morrie J. Early intervention for relapse in schizophrenia: results of a 12-month randomised controlled trial of cognitive behavioural therapy. Psychol Med 2003; 33: 419-431.

(70) Derogatis L, Melisaratos N. The Brief Symptom Inventory: an introductory report. Psychol Med 1983; 13: 595-605.

(71) Birchwood M, Mason R, Macmillan F. Depression, the moralisation and control over psychotic illness. Psychol Med 1993; 23: 387-395. 